\title{
Erratum: On factorisation schemes for the electron parton distribution functions in QED
}

\section{S. Frixione ${ }^{1}$}

INFN, Sezione di Genova,

Via Dodecaneso 33, Genova, I-16146 Italy

PH Department, TH Division, CERN,

Geneva 23, CH-1211 Switzerland

E-mail: stefano.frixione@cern.ch

ERratum to: JHEP07(2021)180

ARXIV EPRINT: 2105.06688

In this erratum we amend eq. (3.7) of ref. [1]. The form reported in the original paper is not the one used in the computations, and in particular it does not lead to the results of sections 4 and 5 (which are correct, and unchanged). The text in the next-to-last paragraph of page 8 of ref. [1] must instead read as follows.

The factorisation-scheme matrix $\mathbb{K}$ of eq. (2.11) is then defined as follows: ${ }^{5}$

$$
\mathbb{K}^{(\Delta)}=\left(\begin{array}{ccc}
K_{e e}^{(\Delta)}(z) & 0 & 0 \\
K_{\gamma e}^{(\Delta)}(z) & 0 & K_{\gamma e}^{(\Delta)}(z) \\
0 & 0 & K_{e e}^{(\Delta)}(z)
\end{array}\right) .
$$

As was discussed in section 2 , the matrix elements of $\mathbb{K}$ whose column index is not equal to the identity of the particle of interest (the electron here, first column) give beyond-NLO effects. By setting them equal to zero as in the second column of eq. (3.7) (that corresponds to the photon), we impose conditions that are identical to what one had to enforce if one were to define a DIS-like scheme for the photon particle, per eqs. (5.42) and (5.47) of ref. [2]. As far as the positron is concerned (third column), its matrix elements are motivated by charge-invariance considerations. Although the positron plays an essentially trivial role here, the form of eq. (3.7) leads to simple analytical computations, in particular to the immediate decoupling of the evolution of the singlet and non-singlet components.

Refs. $[2,3]$ here correspond to refs. $[12,13]$ in the original paper.

\footnotetext{
${ }^{1}$ Now at INFN, Sezione di Genova, Via Dodecaneso 33, Genova I-16146, Italy.

${ }^{5}$ Similarly to what was done in refs. [2,3], in practical applications we limit ourselves to considering the electron, the photon, and the positron.
} 


\section{Acknowledgments}

I am grateful to V. Bertone and G. Stagnitto for discussions that have led to noticing the mistake reported above.

Open Access. This article is distributed under the terms of the Creative Commons Attribution License (CC-BY 4.0), which permits any use, distribution and reproduction in any medium, provided the original author(s) and source are credited.

\section{References}

[1] S. Frixione, On factorisation schemes for the electron parton distribution functions in QED, JHEP 07 (2021) 180 [arXiv:2105.06688] [INSPIRE].

[2] S. Frixione, Initial conditions for electron and photon structure and fragmentation functions, JHEP 11 (2019) 158 [arXiv:1909.03886] [INSPIRE].

[3] V. Bertone, M. Cacciari, S. Frixione and G. Stagnitto, The partonic structure of the electron at the next-to-leading logarithmic accuracy in QED, JHEP 03 (2020) 135 [arXiv:1911.12040] [INSPIRE]. 